\title{
WHAT IS A QUANTUM FIELD THEORY?
}

\author{
BY DAVID C. BRYDGES ${ }^{1}$
}

Introduction. Quantum field theory began in 1927 with a paper by Dirac [1] in which he sought a framework that would unite the classical electromagnetism of Maxwell with quantum mechanics. Ever since then it has been under continuous scrutiny by physicists, which illustrates the fact that in some ways, physics is more highly focused than mathematics. A mathematician is not forced to work on the Riemann hypothesis but ambitious physicists must work in the areas which have experimental durability. In view of all this attention from a different culture, it is not surprising that conventions and modes of reasoning in quantum field theory became difficult for mathematicians to penetrate.

In this article I shall survey a small part of the mathematical work accomplished in quantum field theory. My intent is to convince you that quantum field theories have, in the end, turned out to be very natural generalizations of Brownian motion and other "diffusions" which already occupy a central position in analysis and probability.

I will begin with one-dimensional quantum mechanics and show how there is a diffusion associated with it. In quantum mechanics the object is to determine the wave function

$$
\Psi \equiv \Psi(t, Y) \text {. }
$$

For $t$ fixed, $\Psi$ belongs to $L^{2}(\mathbf{R})$ and is thought of as a time $(t)$ dependent vector in that Hilbert space. Usually, one is given $\Psi$ at some initial time, $t=0$, and the problem is to determine $\Psi$ at a different time by solving the Schrödinger equation

$$
\left(\frac{1}{i} \frac{\partial}{\partial t}-H\right) \Psi=0 .
$$

$H$ is a partial differential operator called the Hamiltonian. It has the form

$$
H=-\partial^{2} / \partial Y^{2}+V
$$

where $V$ denotes multiplication by a function $V(Y)$. Provided some regularity conditions are imposed on $V$ it is possible to solve this equation to obtain a trajectory in $L^{2}(\mathbf{R}), t \rightarrow \Psi(t)$, with value at $t=0$ specified. By considering this process as a map

$$
\Psi(t=0) \stackrel{U_{t}}{\rightarrow} \Psi(t)
$$

Presented to the Society at the Cincinnati meeting, January 15, 1982; received by the editors March 31, 1982.

1980 Mathematics Subject Classification. Primary 81E05, 81E10.

${ }^{1}$ Research partly supported by N.S.F. Grant MCS 79-02490.

(C)1983 American Mathematical Society 0273-0979/82/0000-0616/\$03.50 
we see that at the same time a family $\left\{U_{t}: t \in \mathbf{R}\right\}$ of linear operators on $L^{2}(\mathbf{R})$ is determined. The right way to think of these is suggested by the formula

$$
U_{t}=\exp (i t H) \text {. }
$$

If $H$ were a selfadjoint operator on a finite-dimensional Hilbert space, i.e. a Hermitian matrix, we would know how to understand this using the spectral theorem. Furthermore, $U_{t}$ would be unitary and so $\left\{U_{t}: t \in \mathbf{R}\right\}$ would be a one parameter group of unitary operators. Returning to the Schrödinger equation we insist that the Hamiltonian $H$ obey

(1) $H$ is selfadjoint (on a domain $\mathscr{D}(H) \supset C_{0}^{\infty}$ ),

(2) $H$ is a positive operator: $(\Psi, H \Psi) \geqslant 0$ for all $\Psi$ in the domain of $H$. $\left((\right.$,$) is the inner product on L^{2}(\mathbf{R})$.) The definition of selfadjointness in this context (unbounded densely defined operators on a Hilbert space) is discussed in Volume I of [2]. The principal consequence of this requirement is that the associated $U_{t}$ is unitary (Stone's theorem). This is essential for the physical interpretation of $\Psi$ : namely, $|\Psi(t, Y)|^{2}$ is a probability density and so we want it to be possible to normalize $\Psi$ at $t=0$ so that the total probability $(\Psi, \Psi)$ is 1 and then continue to have this normalization for all time. This norm preservation forces $U_{t}$ to be unitary. A more complete discussion of this point can be found in [3].

Condition (2) is called is called stability of the Hamiltonian. Conditions (1) and (2) are satisfied if the potential $V$ vanishes. Thus they are conditions on $V$.

These two conditions and the spectral theorem for unbounded selfadjoint operators imply that the group $\left(U_{t}\right)$ can be analytically continued in $t$ according to $t \rightarrow i \tau$ with $\tau \geqslant 0$ to yield a contraction semigroup, $\exp (-\tau H)$. Let me explain this a little further: I return to pretending $H$ is a symmetric matrix. The spectral theorem for matrices says that $H$ is unitarily equivalent to a diagonal matrix $\Lambda=\left(\lambda_{n} \delta_{n m}\right)$. Condition (2) implies the eigenvalues $\lambda_{i}$ are positive. By the same unitary equivalence $U_{t}$ becomes the diagonal matrix $\left(\exp \left(i t \lambda_{n}\right) \delta_{n m}\right)$. Under analytic continuation this becomes $\left(\exp \left(-\tau \lambda_{n}\right) \delta_{n m}\right)$ with $\tau \geqslant 0$. Since $\lambda_{n} \geqslant 0$ the entries are bounded by one and it is therefore a contraction (the norm is less than one). Readers familiar with the spectral theorem for unbounded selfadjoint operators will know how to restate this argument in that context.

This associated semigroup $\exp (-\tau H)$ is a diffision semigroup. What does this mean? Let us temporarily set $V=0$. exp it $H$ is an integral operator represented by a kernel $G\left(Y, Y^{\prime}\right)$ which is the fundamental solution of

$$
\left(\frac{1}{i} \frac{\partial}{\partial t}+\frac{\partial^{2}}{\partial Y^{2}}\right) G=0
$$

The kernel of $\exp (-\tau H)$ is the fundamental solution of the diffusion equation:

$$
\left(-\frac{\partial}{\partial \tau}+\frac{\partial^{2}}{\partial Y^{2}}\right) \tilde{G}=0 .
$$

This remark merely defers the question to: why is this called the diffusion equation? I will answer this in the next section by explaining how $\exp (-\tau H)$ is 
connected to a functional integral, an integral over paths which represent possible trajectories of a diffusing particle.

The reason for being interested in the diffusion is that quantum field theories look fairly awesome when approached within the context of quantum mechanics. They are much easier to appreciate in terms of their corresponding diffusions. This was first exploited by Nelson [4] as a mathematical tool. Within the physics literature it has an earlier history attached to Schwinger and Symanzik.

1. Matrices with positive entries, diffusions, and functional integrals. Let $M \equiv\left(M_{i j}\right), i, j \in I$, be a symmetric matrix with zeroes on the diagonal. The resolvent of such a matrix can be represented as a weighted sum over random walks on the index set $I$. To see this, consider the resolvent expansion

$$
(\lambda-M)^{-1}=\frac{1}{\lambda}+\frac{1}{\lambda} M+\frac{1}{\lambda} M \frac{1}{\lambda} M \frac{1}{\lambda}+\cdots .
$$

This expansion converges if $|\lambda|$ is sufficiently large. Consider the $i j$ th entry of the third term

$$
\sum_{k \in I} M_{i k} M_{k j} \frac{1}{\lambda^{3}}
$$

We put the pairs $i k$ and $k j$ in correspondence with the two steps of a random walk, $\omega$, which starts at $i$, goes to $k$, and then to $j$. We can label all the terms coming from the resolvent expansion in this sort of way and obtain a representation for the $i j$ th entry of the resolvent

$$
(\lambda-M)_{i j}^{-1}=\sum_{\omega: i \rightarrow j} J_{\omega} \lambda^{-|\omega|}
$$

where the sum over $\omega$ is a sum over all random walks on $I$ which start at $i$ and end at $j$ after an arbitrary number of steps. $J_{\omega}$ is a weight assigned to $\omega$ by taking the product of entries $M_{l l^{\prime}}$, such that $l l^{\prime}$ is a step in $\omega$ (allowing multiplicity) and $|\omega|$ is the length of $\omega$, the number of steps +1 . This formula is well known in the theory of random walks. It has found some interesting applications in field theory and statistical mechanics [5-7].

Having exhibited the underlying idea, let us now consider the exponential of a matrix $M$ which we split into its diagonal $(D)$ and off diagonal $(O)$ parts, $M=D+O$. By expanding the $2 n$-fold product

$$
e^{t M}=\lim _{n \rightarrow \infty}\left(e^{t D / n} e^{t O / n}\right)^{n}
$$

and putting the sum over matrix indices on the right-hand side in correspondence with random walks we can obtain a representation for the above $2 n$-fold product. However, in this case the additional variable $t$ and the limit $n \rightarrow \infty$ make it convenient to parametrise the random walks by a time $t$, i.e. they are functions $\omega:[0, t] \rightarrow I$. To distinguish this from the situation for the resolvent we call these paths.

If the entries of $O$ are nonnegative, the contribution from each path is positive. This is important in controlling the $n \rightarrow \infty$ limit to obtain 
Proposition. Let $M \equiv\left(M_{i j}\right), i, j \in I$, be a symmetric matrix with nonnegative off diagonal entries. On the space of all paths there is a measure $d \mu^{t}(\omega)$ such that $[\exp (t M)]_{i j}=\int d \mu^{t}(\omega) \delta_{i}(\omega(0)) \delta_{j}(\omega(t))$ where $\delta_{i}(k)$ is the function on $I$ which equals 1 if $k=i$ and zero otherwise.

To see what this has to do with the quantum mechanical semigroup $\exp (-t H)$ notice that the finite difference approximation to the Laplacian $\partial^{2} / \partial Y^{2}$ is the symmetric matrix

$$
\left(\begin{array}{ccccc}
\ddots & & & & \\
1 & -2 & 1 & & \\
& 1 & -2 & 1 & \\
& & 1 & -2 & 1 \\
& & & & \ddots
\end{array}\right) .
$$

Aside from being infinite it is of the form of the $M$ in the proposition above. Furthermore, the finite difference approximation to $H$ is also a matrix which is positive off diagonal.

This suggests that there will be a functional integral representation for $\exp \left(-t\left[-\partial^{2} / \partial Y^{2}+V\right]\right)$. This is correct. If $V=0$ this leads to the Wiener integral and Brownian motion. The following theorem is an extension of these ideas. It is due to Feynman, Kac, and Nelson. This type of theorem is discussed in the new book by Glimm and Jaffe [8]. See also [9].

For this theorem the space of all paths is the set of all continuous functions $\omega: \mathbf{R} \rightarrow \mathbf{R}, \tau \rightarrow \omega(\tau)$.

THEOREM. Let $H=-\partial^{2} / \partial Y^{2}+V$ be selfadjoint with $V$ continous and bounded below. Suppose $\Omega$ is an $L^{2}$ solution of $H \Omega=0$. Then there exists a probability measure $d \mu(\omega)$ on the space of paths such that for $t \in \mathbf{R}^{+}, F, G \in L^{\infty}(\mathbf{R})$, $(F \Omega, \exp (-t H) G \Omega)=\int d \mu F(\omega(0)) G(\omega(t))$.

Note that paths are now functions on the infinite time interval $(-\infty, \infty)$. This is the effect of including $\Omega$. It will allow us to consider $d \mu$ in conjuction with time translations.

In the next section I will examine the inverse question: given a measure on path space, how can one tell if it comes from quantum mechanics and how does one recover quantum mechanics from it? The answer to this will enable us to reconstruct a quantum field theory (to be defined!) from its associated "diffusion".

2. From diffusions back to quantum mechanics. It can be shown that the measure $d \mu$ of the last theorem has two properties:

(1) translation invariance;

(2) "Osterwalder-Schrader Positivity" (O.S. Positivity).

In property (1) the group of translations (time translations) acts on the space of paths according to $\omega(t) \rightarrow \omega_{\tau}(t) \equiv \omega(t+\tau)$. This is a translation by $\tau \in \mathbf{R}$. To understand the O.S. positivity we need some definitions. Let $\left\{t_{1}, t_{2}, \ldots, t_{N}\right\}$ be an arbitrary finite set of positive times. Let $F$ be a continuous function of $N$ 
variables so that we can define a function $F(\omega)$ on paths by

$$
F(\omega) \equiv F\left(\omega\left(t_{1}\right), \omega\left(t_{2}\right), \ldots, \omega\left(t_{N}\right)\right) .
$$

The class of functions obtained in this way forms an algebra, $\mathbb{Q}^{+}$. We can also obtain another algebra $Q^{-}$by taking our finite sets $\left\{t_{1}, \ldots, t_{N}\right\}$ to be negative times.

There is a map

$$
\Theta: \mathbb{Q}^{+} \rightarrow A^{-}
$$

induced by the reflection $t \rightarrow-t$ on $\mathbf{R}$; i.e.,

$$
\Theta F\left(\omega\left(t_{1}\right), \ldots, \omega\left(t_{N}\right)\right) \equiv F\left(\omega\left(-t_{1}\right), \ldots, \omega\left(-t_{N}\right)\right) .
$$

The measure $d \mu$ satisfies

$$
\int d \mu(\omega) \overline{\Theta F} F \geqslant 0
$$

for all (square integrable) $F \in \mathbb{Q}^{+}$. This is known as O.S. positivity.

Conditions (1) and (2) together with technical conditions (e.g. existence of all moments and continuity properties of moments) characterise measures on path space which come from quantum mechanics. Here is a sketch of the proof: consider $f, g \in \mathbb{Q}^{+}$with the "inner product"

$$
(f, g) \equiv \int d \mu \overline{\Theta f} g \text {. }
$$

In fact, it is not necessarily an inner product because $($, ) may be degenerate so we form $\mathbb{Q}^{+} / I$ where $I$ is the ideal of functions $f$ such that $(f, f)=0$. We complete $\mathbb{Q}^{+} / I$ with respect to (, ) to get the "physical Hilbert space", $\mathcal{H}$. On $\mathcal{H}$ one can produce a contraction semigroup $P_{t}$, namely the one induced by time translations. [It is only a semigroup because $\mathbb{Q}^{+}$is not mapped into itself by negative time translation.] $P_{t}$ is easily verified to be selfadjoint relative to $(,$,$) and can be verified to be strongly continuous in t$. A theorem (Hille-Yosida) from functional analysis tells us that such a semigroup can be written in the form

$$
P_{t}=\exp (-t H)
$$

for some selfadjoint positive operator $H$ on $\mathcal{H}$. This is the Hamiltonian. The spectral theorem is then used to produce $\exp (i t H) \equiv U_{t}$ as discussed earlier.

Thus measures $d \mu$ satisfying (1) and (2) and some regularity can be used to construct a Hilbert space, a Hamiltonian $H$, and its associated unitary representation of time translations. There is also a Feynman-Kac-Nelson type formula relating $\exp (-t H)$ and $d \mu$. This reconstruction we have just sketched is set out in detail in [8].

3. Euclidean (Boson) quantum fields. In the last section I was considering measures on the space of all paths in $\mathbf{R}$ with the properties of translation invariance and O.S. positivity. Paths are functions from one variable $t \in \mathbf{R}$ to R. A very natural generalization of this is to make time $\nu$-dimensional, i.e. 
replace the path space of paths $t \rightarrow \omega(t)$ by the "field space" of fields $\phi$,

$$
\phi: \mathbf{R}^{\nu} \rightarrow \mathbf{R} ; \quad x \equiv\left(x_{0}, x_{1}, \ldots, x_{\nu-1}\right) \rightarrow \phi(x) .
$$

We have called our $\nu$-dimensional time $\left(x_{0}, x_{1}, \ldots, x_{\nu-1}\right)$ to suggest "spacetime". If $\nu=1$ we are back to path space and ordinary quantum mechanics.

We now inquire into the existence and properties of measures on field space satisfying the analogues of properties (1) and (2) of the last section. Property (1), translation invariance, becomes Euclidean invariance since this is the group of motions of $\mathbf{R}^{\nu}$ which preserves its standard metric. An element $R$ of the Euclidean group acts on field space by $\phi(x) \rightarrow \phi^{\prime}(x) \equiv \phi(R x)$. In the definition of Osterwalder-Schrader positivity, property (2), we need only replace the reflection $t \rightarrow-t$ appropriate to $\mathbf{R}$ by the reflection $\left(x_{0}, x_{1}, \ldots, x_{\nu-1}\right) \rightarrow\left(-x_{0}, x_{1}, \ldots, x_{\nu-1}\right)$ through the $x_{0}=0$ hyperplane.

Experience has taught us that this framework should be weakened by enlarging field space to $\mathscr{D}^{\prime}\left(\mathbf{R}^{\nu}\right)$, the space of distributions on $\mathbf{R}^{\nu}$. Since $\mathscr{D}^{\prime}$ contains continuous functions we could have done this for $\nu=1$ but the measure produced by the Feynman-Kac-Nelson theorem would be supported only on a small part of $\mathscr{D}^{\prime}$, the continuous functions, which is wasteful. However, for $\nu>1$ it will be necessary to allow fields to be worse behaved. [In all $(\nu>1)$ examples so far constructed continuous fields are a set of measure zero inside $\mathscr{D}^{\prime}$.] I will continue to write equations as if fields were functions. Readers familiar with distributions will know how to make the cosmetic changes required to put in "test functions".

Definition. A $\nu$-dimensional (Boson) Euclidean quantum field theory is a Borel measure $d \mu$ on $\mathscr{W}^{\prime}\left(\mathbf{R}^{\nu}\right)$ satisfying

(1) Euclidean Invariance,

(2) Osterwalder-Schrader Positivity,

(3) Regularity (analyticity and $L^{p}$ bounds for the Fourier transform of $d \mu$ ). (1) and (2) have been explained above. The Euclidean group is the group of motions of $\mathbf{R}^{\nu}$ with its standard metric. It induces an action on functions on $\mathbf{R}^{\nu}$ by change of variables. (This action in turn induces one on $\mathscr{D}^{\prime}$ because ${ }^{2} D^{\prime}$ is dual to $C_{0}^{\infty}\left(\mathbf{R}^{\nu}\right)$.) The property (3) is more technical (and less canonical). It assures that $d \mu$ has moments to all orders and limits the singularities of these moments. We refer the reader to [8] for details.

The next question is what will we get when we try to reconstruct a quantum mechanics in analogy to the path space discussion? In that context recall that invariance of $d \mu$ under translations in time gave rise to a unitary representation $\left[U_{t}=\exp (i t H)\right]$ of the translation group on the physical Hilbert space. Thus in this case it is possible that the whole Euclidean group will, in some form, reappear acting on the physical Hilbert space. The Euclidean group preserves the standard metric $x_{0}^{2}+\cdots+x_{\nu-1}^{2}$ on $\mathbf{R}^{\nu}$. Recall that the construction of the unitary group $\exp (i t H)$ in the path space context involved an analytic continuation $t \rightarrow i t$. The effect of this is to change the Euclidean group into the group of motions of $\mathbf{R}^{\nu}$ with the Lorentz metric $-x_{0}^{2}+x_{1}^{2}+\cdots+x_{\nu-1}^{2}$. This group is called the Poincare group. This is what will appear acting on the physical Hilbert space. 
If we return to the Feynman-Kac-Nelson theorem we see that the Hilbert space associated to $d \mu$, being $L^{2}(\mathbf{R})$, has the distinguished operator of multiplication by $Y$, the coordinate for $\mathbf{R}$. From this one can produce the operator

$$
Y(f) \equiv \int f(t) U_{t} Y U_{-t} d t
$$

for any $f \in C_{0}^{\infty}(\mathbf{R})$. A more detailed study of the abstract reconstruction procedure of the last section shows that there too the Hilbert space has a class of distinguished operators labeled by functions $f \in C_{0}^{\infty}(\mathbf{R})$.

Notice also that the translation group can act on $Y(f)$ in two distinct ways; it can translate the "test function" $f, f(t) \rightarrow f_{\tau}(t) \equiv f(t-\tau)$ or it can act by

$$
Y(f) \rightarrow U_{\tau} Y(f) U_{-\tau} .
$$

However, the two are compatible,

$$
U_{\tau} Y(f) U_{-\tau}=Y\left(f_{\tau}\right)
$$

We refer to this by saying that $Y(f)$ transforms correctly under the translation group.

These remarks should motivate the following theorem which I have lifted from [8]. It was first proved by Osterwalder and Schrader with subsequent work by Fröhlich and Glimm and Jaffe.

THEOREM (RECONSTRUCTION OF MINKOWSKI FIELD THEORIES FROM EUCLIDEAN FIELD THEORIES). Let $d \mu$ be a Borel probability measure on the distribution space $\mathscr{Q}^{\prime}\left(\mathbf{R}^{\nu}\right)$. Suppose it has the three properties, Euclidean invariance, O.S. positivity, and regularity. Then there exists a Hilbert space $\mathcal{H}$ carrying a unitary representation of the Poincare group $\mathcal{P}$. In this space, $\mathcal{H}$, there is a distinguished $\mathcal{P}$ invariant vector $\Omega$, called a (the) vacuum. On $\mathcal{H}$ is defined a class of unbounded selfadjoint operators called field operators $\left\{\phi(f) ; f \in C_{0}^{\infty}\left(\mathbf{R}^{\nu}\right)\right\}$ which satisfy locality and transform correctly under $\mathcal{P}$.

Locality means that $\phi(f)$ and $\phi(g)$ commute when the supports of $f$ and $g$ are space-like separated. The conclusions of this theorem are known as Wightman Axioms [3] for a Minkowski field theory. Thus we have, in the conclusions of this theorem, finally reached a description of what a (Boson) field theory is in the quantum mechanical context as opposed to diffusion context.

There is an important feature of field theories which is not visible in the conclusions of the theorem above: potentially they are simultaneously a theory of discrete objects, "quanta" or "elementary particles". Suppose $d \nu\left(Y_{1}, \ldots, Y_{n}\right)$ is an $n$-dimensional Gaussian measure. Let $\mathfrak{h}$ be the vector space of $n$ vectors $\left\{\left(f_{1}, f_{2}, \ldots, f_{n}\right) ; f_{i} \in \mathbf{R}\right\}$. On $\mathfrak{h}$ define an inner product by

$$
\langle f, g\rangle \equiv \int d \nu Y(f) Y(g) \quad \text { where } Y(f) \equiv \sum_{i}^{n} Y_{i} f_{i} .
$$

Define another Hilbert space, $F \equiv \exp \mathfrak{h} \equiv$ “Fock space”, by

$$
F=\mathbf{R} \oplus \mathfrak{h} \oplus\left(\mathfrak{h} \otimes_{s} \mathfrak{h}\right) \oplus\left(\mathfrak{h} \otimes_{s} \mathfrak{h} \otimes_{s} \mathfrak{h}\right) \oplus \ldots ;
$$


$\otimes_{s}$ denotes the symmetric tensor product. (Yes, it is true that $\exp \left(\mathfrak{h} \oplus \mathfrak{h}^{\prime}\right)=$ $\exp \mathfrak{h} \otimes \exp \mathfrak{h}^{\prime}$.) On $F$ is a distinguished operator, $N$, which equals $j$ times the identity when restricted to the $j$ th summand, $F_{j}$ (starting $j$ at zero). $N$ is called the particle number operator. For each $g \in \mathfrak{h}$ there is an operator $A_{g}^{*}: F \rightarrow F$ defined by setting $A_{g}^{*} \Psi \equiv(\sqrt{n+1}) \times$ symmetrisation of $(\Psi \otimes g)$ for $\Psi \in F_{n}$ and extending in accordance with linearity. Let $A_{g}$ be the adjoint of $A_{g}^{*}$.

Proposition [10]. There is a unitary map $U$ : real $L^{2}\left(\mathbf{R}^{n}, d \nu\right) \rightarrow F$ such that multiplication by $Y(g)$ is exhibited in canonical form

$$
U Y(g) U^{-1}=A_{g}+A_{g}^{*} \text {. }
$$

This theorem about an arbitrary Gaussian measure $d \nu$ can be extended and adapted to show that for the special class of Gaussian field theories (to be discussed further in the next section) the physical Hilbert space $\mathcal{H}$ is isomorphic to a Fock space in terms of which a "particle structure" is defined. For general field theories the situation is more complex and not completely worked out. See [8].

Results on existence. A finite-dimensional Gaussian integral has the property that its moments are all determined by the matrix of second moments; thus if $d \nu$ is the Gaussian measure

$$
d \nu(\mathbf{x})=\left(\prod_{i=1}^{n} d x_{i}\right) \exp \left(-\frac{1}{2} \sum_{i, j} x_{i} A_{i j} x_{j}\right) / \text { Normalization }
$$

and, if $S$ is a set of indices, then

$$
\int d \nu(\mathbf{x}) \prod_{i \in S} x_{i}=\sum_{\gamma} \prod_{i, j \in \gamma} \int d \nu(x) x_{i} x_{j}
$$

where $\gamma$ is summed over all partitions of $S$ into pairs. In fact, if the moments of a probability measure $d P$ in $\mathbf{R}^{n}$ obey such a formula, $d P$ is Gaussian. For a measure $d \mu$ on a function space the analogue of this formula is

$$
\int d \mu(\phi) \prod_{x \in S} \phi(x)=\sum_{\gamma} \prod_{x, y \in \gamma} \int d \mu(\phi) \phi(x) \phi(y)
$$

where $S$ is a finite set of points in $\mathbf{R}^{\nu}$. (For $\mathscr{Q}^{\prime}$, points must be replaced by test functions.) If $d \mu$ satisfies such an identity for all $S$ we say $d \mu$ is Gaussian.

Given knowledge of Minlos' theorem $[9,10]$ it can be shown that Gaussian Euclidean quantum field theories exist for all spacetime dimensions $\nu$. The reconstruction theorem provides corresponding Minkowski field theories which describe noninteracting elementary particles. Thus the existence problem is to show that there are non-Gaussian Euclidean quantum field theories. This has been done in spacetime dimensions $\nu=1,2,3$ [8]. For $\nu \geqslant 5$ techniques are now being developed to prove nonexistence of non-Gaussian field theories. I will discuss some such results momentarily. $\nu=4$ is evidently some kind of borderline. Right now the expectation is that a special class of quantum field theories-nonabelian gauge theories - can be non-Gaussian in 4 dimensions and probably no other class yet studied can do likewise. This is the expectation 
of the physicists (whose batting average is very good!). No interesting nonabelian field theories have yet been constructed. There has been some progress on abelian gauge theories. For a good review of these directions, see [11].

4. Construction and renormalization. Let $S(\phi)$ be the functional

$$
S(\phi) \equiv \frac{Z}{2} \int[\nabla \phi(x)]^{2} d^{\nu} x+\int P(\phi(x)) d^{\nu} x
$$

where $P$, known as the "interaction", is a polynomial in $\phi$, bounded below and $Z \geqslant 0$. If $\phi^{\prime}(x)$ is obtained from $\phi(x)$ by a Euclidean change of coordinates then $S\left(\phi^{\prime}\right)=S(\phi)$. Our first attempt to write down a Euclidean invariant measure $d \mu$ on a function space might be to associate to each point $x$ in spacetime a random variable $\phi(x)$ and let the collection $\phi=\left\{\phi(x)=x \in \mathbf{R}^{\nu}\right\}$ be distributed according to

$$
\text { “ } d \mu(\phi) \equiv e^{-S(\phi)} \prod_{x \in \mathbf{R}^{\nu}} d \phi(x) / \text { Normalization”. }
$$

This is a meaningless equation for several different reasons. In particular, the uncountable product of Lebesgue measures $d \phi(x)$ is incomprehensible. Nevertheless, it suggests the following procedure: make a finite difference approximation by selecting a finite lattice $L \subset \mathbf{R}^{\nu}$ and define the finite-dimensional probability measure

$$
d \mu_{L}(\phi) \equiv e^{-S_{L}(\phi)} \prod_{x \in L} d \phi(x) / \text { Normalization, }
$$

where $S_{L}(\phi)$ is the appropriate finite difference approximation to $S(\phi)$. This fails to be Euclidean invariant because the lattice is not invariant. However if the lattice is symmetric about the hyperplane $x_{0}=0$ it is O.S. positive. This is not too difficult to prove especially in the special case $\nu=1$.

Next we attempt to recover Euclidean invariance. Choose a sequence of lattices $L_{1} \subset L_{2} \subset \cdots$, getting larger and finer so as to approximate $\mathbf{R}^{\nu}$. We define functions (actually distributions) by

$$
G\left(x_{1}, \ldots, x_{N}\right) \equiv \lim _{i \rightarrow \infty} \int d \mu_{L_{i}}(\phi) \phi\left(x_{1}\right) \cdots \phi\left(x_{N}\right)
$$

if it exists. Next we attempt to find a measure $d \mu$ on $\mathscr{Q}^{\prime}\left(\mathbf{R}^{\nu}\right)$ whose moments are $G\left(x_{1}, \ldots, x_{N}\right), x_{1}, \ldots, x_{N}$ and $N$ arbitrary, i.e. $d \mu$ is such that as distributions

$$
\int_{\mathscr{D}^{\prime}} d \mu(\phi) \phi\left(x_{1}\right) \cdots \phi\left(x_{N}\right)=G\left(x_{1}, \ldots, x_{N}\right) .
$$

(This is a generalized Hausdorff moment problem.)

In doing all this the existence of the limit is not the worst problem, especially if one is willing to use compactness and subsequences. The real problems center around whether the limiting measure is trivial (zero or Gaussian). To prevent this possibility it is necessary (for $\nu>1$ ) to allow the parameters, $Z$ and the coefficients in the polynomial $P$, to vary as the limit $i \rightarrow \infty$ is taken. This is called renormalization. The parameters are known as "bare coupling 
constants". Some of them have to be chosen to diverge to $\infty$ as $i \rightarrow \infty$. A relatively simple discussion of a renormalization will become available in [12].

This type of construction yields the " $P(\phi)$ " " field theories. Gauge theories result from a different choice of functional $S$. We replace $\nabla$ by covariant gradient acting on section $\phi(x)$ and we also integrate over the associated connections.

My final remarks concern the recent theorem of Aizenman [13] and further developments by Fröhlich [5]. Aizenman has shown that for spacetime dimensions $\nu \geqslant 5$, and

$$
P(\phi)=a \phi^{2}+\lambda \phi^{4}, \quad a \in \mathbf{R}, \lambda \geqslant 0,
$$

that there is no way to choose lattice dependent bare couplings $Z, a, \lambda$ to yield a non-Gaussian field theory. Fröhlich has extended this to a theorem in four dimensions with the additional hypothesis $Z_{L} \rightarrow 0$. These are the beginnings of a "no go" theory for field theories in $\nu \geqslant 5$ (4?!) dimensions. Some partial results of this type were previously obtained by Glimm and Jaffe [8]. In the physics literature there is a fairly convincing heuristic explanation for this and other facts(?) but mathematical work on it has barely begun.

ACKNOWLEDGEMENT. This area of research is largely the creation of Wightman, Glimm, Jaffe and Nelson. Other major contributions came from Simon, Spencer and Fröhlich. My own understanding has been greatly enhanced by conversation with Glimm, Federbush, Fröhlich and Spencer.

\section{REFERENCES}

1. P.A. M. Dirac, Proc. Roy. Soc. 114 (1927). See also J. von Neumann, Mathematical foundations of quantum mechanics, Princeton Univ. Press, Princeton, N. J., 1955.

2. M. Reed and B. Simon, Methods of modern mathematical physics, Volumes I, II, Academic Press, New York, 1972, 1975. 1964.

3. R. F. Streater and A. S. Wightman, PCT spin \& statistics and all that, Benjamin, New York,

4. E. Nelson, Construction of quantum fields from Markov fields, J. Funct. Anal. 12 (1973), 97-112 and $A$ quartic interaction in two dimensions, Mathematical Theory of Elementary Particles (R. Goodman and I. Segal (eds.)), MIT Press, Cambridge, Mass., 1966.

5. J. Fröhlich, On the triviality of $\lambda \phi_{d}^{4}$ theories and the approach to the critical point in $d(\geqslant) 4$ dimensions, Inst. Hautes Études Sci., preprint. See also [13].

6. D. Brydges, J. Fröhlich and T. Spencer, The random walk representation of classical spin systems and correlation inequalities, Comm. Math. Phys. 83 (1982), 123.

7. D. Brydges and P. Federbush, A lower bound for the mass of a random Gaussian lattice, Comm. Math. Phys. 62, 79 (1978).

8. J. Glimm and A. Jaffe, Quantum physics, a functional integral point of view, Springer-Verlag, Berlin and New York, 1981. See also [10].

9. G. Simon, Functional integration and quantum physics, Academic Press, New York, 1979.

10. 1974. , The $P(\phi)_{2}$ Euclidean quantum field theory, Princeton Univ. Press, Princeton, N. J.,

11. E. Seiler, Gauge theories as a problem of constructive quantum field theory and statistical mechanics, Troisième Cycle de la Physique Lecture Notes, 1981; Springer-Verlag, Berlin and New York (to appear).

12. D. Brydges, J. Fröhlich and A. Sokal, A new construction of $\phi_{3}^{4}$ (in preparation).

13. M. Aizenman, Proof of the triviality of $\phi_{d}^{4}$ field theory and some mean field features of Ising models for $d>4$, Phys. Rev. Lett, 47, 1 (1981).

Department of Mathematics, University of Virginia, Charlottesville, Virginia 22903 